\title{
Dirección escolar y liderazgo pedagógico: un análisis de contenido del discurso de los directores de centros educativos en la Comunidad Autónoma de Andalucía (España)
}

\author{
Maximiliano Ritacco Real ${ }^{1}$ \\ Francisco Javier Amores Fernández²
}

\section{Resumen}

Investigaciones de índole nacional e internacional resaltan la mejora de los resultados de aprendizaje del alumnado cuando, entre otros aspectos, la asesoría y el grado de autonomía en lo pedagógico de la dirección escolar es real. No obstante, en España, el ejercicio del liderazgo no implica el desarrollo pleno de su dimensión pedagógica. La complejidad y la inercia tradicional de la cultura escolar de los centros inciden de forma negativa en estas cuestiones. De corte cualitativo e interpretativo, el estudio ha indagado en las perspectivas y valoraciones de quince directores acerca del ejercicio de su liderazgo y de las posibilidades para dęsarrollar un liderazgo pedagógico. La aplicación de la técnica del análisis de contenido dio como resultado la consolidación de tres categorías generales: a) visiones; b) resistencias y c) requerimientos. De cara a la fase interpretativa, la organización emergente de sus sub-categorías y el establecimiento de un índice de frecuencia categorial (IFC), como ingrediente cuantitativo, permitieron completar el grado de compresión y especificación de la información.

\section{Palabras clave}

Liderazgo escolar - Liderazgo pedagógico - Dirección escolar - Mejora escolar Organización escolar.

\section{School management and pedagogical leadership: a content analysis of the schools principals discourse in the Autonomous Community of Andalusia (Spain)}

\section{Abstract}

National and international investigations highlight the improvement of student learning outcomes when, among other aspects, the advice and the pedagogical autonomy degree of the school principals are real. However, in Spain, the exercise of leadership does not imply the full development of their pedagogical dimension. The complexity and the traditional

\footnotetext{
1- Universidad de Zaragoza. Teruel, España. Contacto: ritacco@unizar.es

2- Universidad de Cádiz. Cádiz, España. Contacto: franciscojavier.amores@uca.es

DOl: http://dx.doi.org/10.1590/S1678-4634201709162034
} 
inertia of the school culture impact these issues in a negative way. With a qualitative and interpretative approach, the study has investigated the prospects and the valuation of fifteen directors about the exercise of leadership and the possibilities of developing pedagogical leadership. The application of the technique of content analysis resulted in the consolidation of three general categories: to) visions; (b) resistance; and (c) requirements. Facing the interpretation phase, the emerging organization of its sub-categories and the establishment of a categorical frequency index (CFI), as a quantitative ingredient, allowed us to complete the compression and specification of the information.

\section{Keywords}

School leadership - Pedagogical leadership - School improvement - School management - School organization.

\section{Introducción}

El presente estudio ha sido subvencionado por el Ministerio de Ciencia y Tecnología (VI Plan Nacional de Investigación Científica, Desarrollo e Innovación Tecnológica -I+D+I-) formando parte del proyecto de investigación titulado Liderazgo centrado en el aprendizaje y su impacto en la mejora: Prácticas y resultados en Secundaria. A su vez, se vincula al strand (1) Directores de escuela exitosos del Proyecto internacional de directores de escuela exitosos, y se integra en la Red de Investigación sobre Liderazgo y Mejora Educativa (RILME).

Para el diseño de la investigación nos adjuntamos a las recomendaciones del Informe Mejorando el liderazgo escolar elaborado por la Organización para Cooperación y Desarrollo Económico (OCDE, 2011). Su enfoque sitúa a la escuela en un lugar clave. Se comprende que si bien está inmersa en la complejidad de los procesos de globalización (movilidad, temporalidad, tecnología, etc.), no solo debe responder a las demandas del mercado laboral, sino también, a las expectativas socio-políticas de ofrecer una educación de calidad para todo el alumnado.

En este marco, la OCDE (2011) y la Comisión Europea $(2008$; 2009) aconsejan la búsqueda de prácticas innovadoras que eleven los resultados de aprendizaje. Atendiendo a ello, autores como Murillo (2003) o Bolívar (2012; 2014a) señalaron la importancia de los movimientos mejora de la escuela y de reestructuración escolar como telón de fondo a la propuesta de las comunidades profesionales de aprendizaje. En estos casos, las dinámicas colaborativas y de trabajo en equipo son sustentadas por un liderazgo distribuido que consigue generar confianza y responsabilidad en el personal en una sola dirección, la mejora del desarrollo educativo (BOLAM, et al., 2005; HARGREAVES; FULLAN, 2014; VAILLANT, 2015; VAILLANT; RODRÍGUEZ, 2016; WEINSTEIN; HERNÁNDEZ, 2015).

Al respecto, una revisión de los principales indicadores de cambio recomendados (ROWAN, 1990; MAYNTZ, 2001; FULLAN, 2002; ELMORE, 2010; FERNANDA; ZAMBÃO, 
2015) nos señala la necesidad de mayor autonomía por parte de los centros educativos. Gestionar el currículum, capacitación profesional, innovación, etc., deben ser prácticas integradas en los centros, no impuestas, que impliquen el apoyo y la dotación de recursos por parte de la administración educativa.

Desde una perspectiva sistémico-organizativa, el modelo que se instaura como el más idóneo es el post-burocrático (MAROY, 2009; BOLÍVAR, 2010; 2012; MASON, 2011). Postula desarrollar la capacidad pedagógica del profesorado aumentando sus cuotas de autonomía. Se comprende pues que la (des) regulación post-burocrática ha supuesto un proceso de reconfiguración de la dinámica inter-niveles del sistema educativo (macro, meso y micro) abriendo un conjunto de ámbitos de autonomía, entre ellos, el de la dirección escolar (BARROSO, 2006).

Así mismo, se fortalece el ejercicio de un liderazgo pedagógico en términos de crear las condiciones adecuadas para el aprendizaje y el desarrollo profesional docente. Bajo este marco, el informe McKinsey (BARBER; MOURSHED, 2007), ha constatado el efecto director como el segundo factor (interno-indirecto) de influencia a la hora de elevar los resultados educativos del alumnado.

Desde este enfoque, si bien continúa siendo el director el núcleo del liderazgo formal, se recalca que la mejora se incrementa a medida que distribuye su liderazgo (SPILLANE, 2006). En esta línea se hace atrayente la idea del liderazgo distribuido que anima al director a potenciar el liderazgo de los docentes e invita, mediante dinámicas colaborativas y el trabajo en equipo, a la ruptura con la centralidad rígida y estática de la cultura escolar de antaño. En palabras de Bolívar: "El liderazgo pedagógico se entiende como un grupo de gente que aprende y trabaja junta en una comunidad profesional de aprendizaje con un sentido de propósito compartido" (2012, p. 257).

No obstante, cabe resaltar que en estructuras débilmente articuladas, fragmentadas y/o atomizadas, la resistencia del profesorado a este tipo de procesos suele ser prominente. Se depende, en este sentido, del voluntarismo y la confianza debido a las escasas posibilidades de supervisión y evaluación de la tarea docente (RITACCO; AMORES; MORAL, 2015).

Asociando la perspectiva del liderazgo pedagógico al estado de la cuestión de la dirección escolar en España, el panorama dista de cubrir las expectativas. Los remanentes del modelo administrativista-burocrático sitúan al caso español en un momento de larga transición que partió a mediados de los años setenta con el reclamo de instauración del modelo electivo de dirección (Consejo escolar y profesorado). En la actualidad todavía se debate, no solo este aspecto, sino también:

a) la dicotomía de los directores son profesores;

b) el itinerario transeúnte en el cargo (ida y vuelta, docente-director-docente);

c) el ejercicio limitado por el tiempo (períodos de cuatro años);

d) la falta de autonomía en decisiones sobre la enseñanza y currículum;

e) la debilidad estructural en el diseño de entornos de mejora;

f) las escasas atribuciones para ejercer la dimensión pedagógica;

g) el peso de las tareas administrativas y de gestión. 
Todas estas cuestiones, como es de esperarse, tienen un impacto en el desarrollo educativo del alumnado (BOLÍVAR et al., 2004).

No obstante, una serie de cambios legislativos abren la puerta a nuevas orientaciones para la dirección escolar en España. Como parte del proceso de reforma de la gobernanza y regulación de la educación pública, la Ley Orgánica de Educación (ESPAÑA, 2006) marcó un punto de partida. La búsqueda de un perfil colegiado de dirección, el incremento de descentralización y autonomía pedagógica, el aumento del grado de responsabilidad por los resultados de aprendizaje, la búsqueda de itinerarios de profesionalización directiva y/o des-corporativizar el sistema de elección fueron algunas de las iniciativas marcadas.

En el terreno de la práctica, a la hora de materializar estas cuestiones, no tardaron en identificarse una serie de resistencias. Entre los estudios realizados dentro del marco del proyecto $\mathrm{I}+\mathrm{D}$, destacamos aquellos que reflejan como las inercias tradicionalistas de la cultura escolar de los centros educativos enlentecen o impiden el proceso de cambio y mejora (MORAL; AMORES, 2014; MORAL; AMORES; RITACCO, 2016).

Los aportes investigativos han reflejado como la escena rígida y fragmentada de las dinámicas organizativas de los centros reducen el margen de acción de los directores hacia el aislamiento en pos del cumplimiento, mayormente, de tareas administrativas y burocráticas (GIMENO et al., 1995; BOLÍVAR et al., 2004; MORAL; AMORES, 2014).

Por otro lado, la cuestión de la autoridad formal y/o moral de los directores (RITACCO; AMORES; MORAL, 2015) y la ausencia de una regulación normativa que empodere en la toma de decisiones acerca de aspectos pedagógicos o de innovación educativa, centran los canales de acción en la necesidad del apoyo del claustro (profesorado, padres, administración). En este punto, entran en juego las habilidades sociales y de relación del director. Por medio del convencimiento y la negociación es posible generar un capital relacional con el personal que le permitiría sacar adelante sus propuestas. En gran medida, estamos refiriendo a un tipo de autoridad (moral) que se construye a largo plazo y que se diluye en cuanto se releva el cargo directivo.

No menos importante es la resistencia docente a la supervisión de sus funciones (DOMINGO; RITACCO, 2015). Con la posibilidad de blindarse en su situación funcionarial y en un sistema de promoción que valora la antigüedad en el cargo por encima de la capacitación profesional (o los resultados de aprendizaje del alumnado), el profesorado orienta sus expectativas hacia la movilidad y/o aproximación al lugar de procedencia. En este sentido, una vez conseguido el destino, la motivación por la mejora tiende a decrecer, mientras que la tarea docente tiende a atomizarse.

Teniendo en cuenta el panorama y en orden de reconfigurar el status de la dirección escolar en España, no son pocas las propuestas en términos de objetivos de mejora en el planteamiento de la función directiva (LÓPEZ YAÑEZ; LAVIÉ, 2010; BOLIVAR, 2012; MORAL; AMORES, 2014). Entre ellas podríamos mencionar: a) lograr una mayor capacidad en la gestión del personal; b) aumentar la potestad disciplinaria; c) incrementar su profesionalización y formación específica; d) responder a la necesidad de apoyos y recursos suplementarios (supervisores, asesores); y e) fortalecer la capacidad de intervención pedagógica (metodologías y procesos de enseñanza eficaces). 
Sin más, para la comprensión de esta situación se consideran necesarios estudios que recojan las perspectivas y valoraciones en primera persona de aquellos que construyen los espacios y contextos referidos. El discurso y análisis de sus vivencias pueden ayudar a definir con más claridad la radiografía y la interpretación de los procesos que intervienen en la búsqueda del desarrollo del liderazgo pedagógico en la dirección escolar en España.

\section{Metodología}

La metodología del estudio se inscribe dentro del enfoque y/o paradigma interpretativo de investigación (KOETTING, 1984; POPKEWITZ, 1988). Desde esta perspectiva se configura el diseño de nuestra investigación estableciendo una progresión de objetivos metodológicos (Tabla 1).

Atendiendo a los objetivos propuestos y a las fuentes testimoniales de los datos (biográfico-narrativa), se consideró el alto grado de idoneidad de una metodología de investigación de corte cualitativo (STRAUSS; CORBIN, 2002; FLICK, 2007; SMITH; FLOWER; LARKIN, 2009). En esta línea, se consolida la idea de aplicar la técnica del análisis de contenido como base para la reducción y estructuración de la información (KRIPPENDORF, 1990; PORTA; SILVA, 2003; ÁVILA DE LIMA, 2013). La prestación del análisis de contenido se tradujo, mayormente, en la aproximación inductiva-inferencial realizada a partir de los datos verbales, simbólicos y comunicativos. Presente casi al completo en el proceso de categorización de los datos, hizo posible integrar ciertos matices de corte cuantitativo referentes al recuento (frecuenciación) de los indicadores extraídos del discurso. Admitió, por último, en la fase comprensiva e interpretativa, establecer un contínuum con la profundización sucesiva de la teoría fundamentada (STRAUSS; CORBIN, 2002; CHARMAZ, 2004; WERTZ; CHARMAZ; MCMULLEN, 2011; BROOKS; NORMOORE, 2015).

Tabla 1 - Objetivos metodológicos del estudio. Nivel de concreción.

\begin{tabular}{|c|c|c|}
\hline Objetivos & & Nivel de concreción \\
\hline Objetivo 1 & $\begin{array}{l}\text { Captar, analizar y organizar la información emergente de los testimonios recogidos } \\
\text { en el trabajo de campo. }\end{array}$ & $\begin{array}{l}\text { Nivel } 1 \\
\text { Análisis de contenido }\end{array}$ \\
\hline Objetivo 2 & $\begin{array}{l}\text { Objetivar las creencias, percepciones y valoraciones, elementos no observables de la } \\
\text { conducta. }\end{array}$ & \multirow{2}{*}{$\begin{array}{l}\text { Nivel } 2 \\
\text { Análisis de contenido }\end{array}$} \\
\hline Objetivo 3 & Profundizar en el entendimiento del significado de las (inter)acciones de los sujetos. & \\
\hline Objetivo 4 & $\begin{array}{l}\text { Comprender las subjetividades emergentes de la indagación acerca del liderazgo } \\
\text { escolar y el desarrollo del liderazgo pedagógico en los centros educativos. }\end{array}$ & $\begin{array}{l}\text { Nivel } 3 \\
\text { Teoría fundamentada }\end{array}$ \\
\hline
\end{tabular}

Fuente: Elaborado por los autores. 
Como se observa, nos adjuntamos a una combinación paradigmática que supera el simplismo de la polémica que fragmenta el modelo metodológico cuantitativo del cualitativo (GUBA; LINCOLN, 1994). Pragmáticamente, este aspecto, si bien eleva el grado de complejidad metodológica del estudio, legitima una concepción plural que amplía las posibilidades epistemológicas de la investigación.

\section{La muestra de informantes y centros educativos}

Para conformar la muestra del estudio se seleccionaron quince centros de educación secundaria de la comunidad autónoma de Andalucía (España) considerando: a) que estén financiados con fondos públicos; b) su indice socio-económico y cultural -ISEC3-(cinco ISEC alto, cinco ISEC medio y cinco ISEC bajo); c) la oferta educativa de los centros (planes y programas) y d) su ubicación geográfica, (cinco en el centro urbano, cinco en barrios aledaños al macro-centro y cinco en zonas periféricas y comarcales).

Delimitada la muestra de centros se avanzó sobre aquellos criterios que perfilarían a los sujetos informantes, al respecto: a) que hayan ocupado, al menos, un período completo en el cargo directivo (cuatro años); b) que puedan brindar una mirada procesual y cronológica de su experiencia en la dirección; c) que posean una trayectoria de innovaciónen la función directiva; y d) que hayan formado parte de procesos de mejora educativa.

Tabla 2 - Criterios de selección y distribución de la muestra de informantes y centros ${ }^{4}$.

\begin{tabular}{|c|c|c|c|c|c|c|c|c|}
\hline \multicolumn{4}{|c|}{ Informantes } & Centros & Ubicación y & Índice socio, & Oferta & Financiación \\
\hline \multirow{3}{*}{$\begin{array}{c}1 \text { Cargo } \\
\text { 4años }\end{array}$} & \multirow{3}{*}{$\begin{array}{c}\text { Persp. } \\
\text { procesual }\end{array}$} & \multirow{3}{*}{$\begin{array}{c}\text { Trayectoria } \\
\text { de } \\
\text { innovación }\end{array}$} & \multirow{3}{*}{$\begin{array}{l}\text { Proceso } \\
\text { de } \\
\text { mejora }\end{array}$} & C11-C15 & $\begin{array}{c}\text { Zonas } \\
\text { periféricas } \\
\text {-comarcales }\end{array}$ & $\begin{array}{l}\text { ISEC } \\
\text { Bajo }\end{array}$ & \multirow{2}{*}{$\begin{array}{l}\text { Educación } \\
\text { secundaria } \\
\text { Bachillerato }\end{array}$} & \multirow{3}{*}{$\begin{array}{l}\text { Fondos } \\
\text { públicos }\end{array}$} \\
\hline & & & & C6 - C10 & $\begin{array}{c}\text { Macro centro } \\
\text { urbano }\end{array}$ & $\begin{array}{l}\text { ISEC } \\
\text { Medio }\end{array}$ & & \\
\hline & & & & $\mathrm{C} 1-\mathrm{C} 5$ & $\begin{array}{l}\text { Centro } \\
\text { urbano }\end{array}$ & $\begin{array}{l}\text { ISEC } \\
\text { Alto }\end{array}$ & $\begin{array}{c}\text { Programas de } \\
\text { atención a la } \\
\text { diversidad }\end{array}$ & \\
\hline
\end{tabular}

Fuente: Elaborado por los autores.

\section{Técnicas de recogida y procesamiento de datos}

Se realizaron quince entrevistas biográficas en profundidad, no estructuradas, con la intención de obtener una descripción situacional clara de los contextos y lograr interpretaciones fidedignas del significado que tienen los fenómenos descritos por los directivos escolares (VALLÉS, 2009).

3- La Consejería de Educación (Junta de Andalucía) a través de la Agencia Andaluza de Evaluación Educativa (2006-2011) establece considerar el ISEC de los centros pro-formado a partir de los cuestionarios de contexto de familia que integra variables tales como: recursos de hogar, nivel de estudios, ocupación de los padres, etc.

4- Las entrevistas se realizaron entre noviembre y junio del 2015 en centros educativos de educación secundaria (financiados con fondos públicos) de la Comunidad Autónoma de Andalucía. 
Acorde con los objetivos propuestos se diseñó un guión de la entrevista considerando un conjunto de ámbitos temáticos: a) el desarrollo del liderazgo escolar (funciones, tareas, intervención); b) el desarrollo del liderazgo pedagógico y sus capacidades (labor pedagógica y trasnformacional); y c) el impacto de su tarea directiva en los resultados de aprendizaje del alumnado.

Otros aspectos de interés de la entrevista se pueden observar en la Tabla 3:

Tabla 3 - Aspectos complementarios en la realización de la entrevista.

\begin{tabular}{|c|c|c|c|}
\hline Centro educativo & Tiempo de entrevista & Sexo & Edad \\
\hline C1 & $01: 59: 47$ & V & 63 \\
\hline $\mathrm{C} 2$ & $01: 14: 52$ & $M$ & 56 \\
\hline C3 & $01: 14: 47$ & V & 45 \\
\hline C4 & $00: 42: 14$ & V & 37 \\
\hline $\mathrm{C5}$ & $00: 58: 59$ & $M$ & 34 \\
\hline C6 & $01: 22: 36$ & V & 56 \\
\hline $\mathrm{C} 7$ & 01:19:53 & M & 38 \\
\hline $\mathrm{C} 8$ & $01: 29: 43$ & V & 54 \\
\hline $\mathrm{Cg}$ & 01:06:28 & $\mathrm{M}$ & 45 \\
\hline C10 & 01:09:47 & V & 44 \\
\hline $\mathrm{C} 11$ & $00: 53: 34$ & V & 49 \\
\hline $\mathrm{C} 12$ & 00:58:05 & V & 39 \\
\hline $\mathrm{C} 13$ & 01:18:12 & V & 45 \\
\hline $\mathrm{C14}$ & $01: 05: 49$ & $M$ & 46 \\
\hline $\mathrm{C15}$ & 01:29:19 & V & 38 \\
\hline Total & $18: 15: 33$ & $M=5-V=10$ & 689 \\
\hline Promedio & 01:13:34 & $1 \times 3$ son mujeres & 46 años \\
\hline
\end{tabular}

$M=$ mujer. $V$ = varón. $C$ = Centro Educativo.

Fuente: Elaborado por los autores.

Proceso de análisis y estructuración de los datos: aplicación del análisis de contenido

Una vez realizado el trabajo de campo se procedió a la reducción y categorización de los datos por medio de la aplicación de la técnica del análisis de contenido -Tabla 4(KRIPPENDORF, 1990; PORTA; SILVA, 2003; ÁVILA DE LIMA, 2013; WERTZ; CHARMAZ; MCMULLEN, 2011; BOLÍVAR, 2014b).

En su primera fase, fue de gran importancia establecer una nomenclatura de codificación, así como, que el procedimiento de transcripción respete el canal y las fuentes de información (WERTZ; CHARMAZ; MCMULLEN, 2011). Una vez en el análisis, el intercambio dialéctico constante entre el fundamento teórico y la información recogida permitieron la identificación progresiva, en el texto, de unidades de registro (UR).

La segunda fase, se identificó por el $1^{\text {er }}$ vuelco de las UR extraídas de las fuentes de información fueron organizadas en una serie de categorías que atendieron a 
similitudes, asociaciones y representaciones soportadas por el estudio de las temáticas de la investigación. En este punto, las unidades de registro cambian su nomenclatura a unidades de análisis (UA) o indicadores (I).

Dado el bajo nivel de concreción de las categorías emergentes, se avanza hacia una tercera fase de la estructuración de los datos. En sintonía, aumentando el nivel de especificación, se realizó un análisis de las categorías que finaliza en un $2^{\circ}$ vuelco de las UA y la consecuente reorganización de sus sub-categorías.

Una cuarta fase (final), se caracterizó por asignar un índice de frecuencia categorial (IFC) a cada categoría y sub-categoría. El IFC se define por el recuento de indicadores (I). Cabe aclarar que desde una perspectiva interpretativa el IFC no es estadístico, sino aproximativo ya que representa el peso significativo de la información contenida en las UA o I. En este estudio, el IFC será porcentual, dado que facilita una interpretación proporcional en función del IFC total de las categorías superiores.

Tabla 4 - Fases del análisis de contenido. Categorías y sub-categorías emergentes.

\begin{tabular}{|c|c|c|}
\hline \multicolumn{3}{|c|}{ Análisis de contenido } \\
\hline \multicolumn{3}{|c|}{$\downarrow$ Transcripción y codificación (x 15 entrevistas) $\downarrow$} \\
\hline \multicolumn{3}{|c|}{$\downarrow$ Identificación de unidades de registro -UR- $\downarrow$} \\
\hline \multicolumn{3}{|c|}{$\downarrow 1^{\text {er }}$ vuelco de UR $\downarrow$} \\
\hline \multicolumn{3}{|c|}{$\begin{array}{c}\text { Categorías } \\
\text { (Unidades de Análisis -UA-) }\end{array}$} \\
\hline $\begin{array}{l}\text { Visión del liderazgo } \\
\text { Pedagógico }\end{array}$ & $\begin{array}{c}\text { Requerimientos para } \\
\text { un liderazgo pedagógico }\end{array}$ & Resistencias al liderazgo pedagógico \\
\hline \multicolumn{3}{|c|}{$\downarrow 2^{\circ}$ Vuelco de Unidades de Análisis (UA) $\downarrow$} \\
\hline \multicolumn{3}{|c|}{ Sub-categorías } \\
\hline $\begin{array}{l}\text { Visión liderazgo } \\
\text { Pedagógico }\end{array}$ & $\begin{array}{c}\text { Requerimientos liderazgo } \\
\text { Pedagógico }\end{array}$ & $\begin{array}{l}\text { Resistencias liderazgo } \\
\text { Pedagógico }\end{array}$ \\
\hline $\begin{array}{l}\text {-Trabajo en equipo } \\
\text {-Resultados de aprendizaje } \\
\text {-Establecer directrices comunes }\end{array}$ & $\begin{array}{c}\text {-Capacidad pedagógica } \\
\text {-Organización-coordinación } \\
\text {-Perspectiva del centro y contexto } \\
\text {-Apoyo del claustro } \\
\text {-Facilitador } \\
\text {-Capacidad de intervención }\end{array}$ & $\begin{array}{c}\text {-Modelo de dirección } \\
\text {-Formación específica } \\
\text {-Gestión del profesorado } \\
\text {-Burocracia } \\
\text {-Formación pedagógica-Profesorado } \\
\text {-Situación jerárquica-autoridad } \\
\text {-Autonomía }\end{array}$ \\
\hline
\end{tabular}

$\uparrow$ Índice de Frecuencia Categorial (IFC) -porcentual- $\uparrow$

Fuente: Elaborado por los autores.

\section{Análisis de los resultados}

Tal como se esquematiza en la Tabla 4 las categorías emergentes del análisis hacen referencia al ejercicio de la dirección escolar en función del desarrollo del liderazgo pedagógico. En este sentido, se observa una estructura categorial que otorga coherencia a los resultados del estudio: a) la necesidad de integrar una visión (perspectiva previa) 
para alcanzar el liderazgo pedagógico; [cumpliendo con unos] b) requerimientos para alcanzarlo; [y enfrentándose] c) a unas resistencias para poder desarrollar la capacidad pedagógica del liderazgo.

Teniendo en cuenta el comportamiento del peso de la información (Gráfico 1), la distribución del índice de frecuencia categorial (IFC porcentual) revela una tendencia preocupante: más de la mitad del total de los indicadores (I) se agrupan en la categoría resistencias (55,6 IFC\%), seguida de la categoría requerimientos $(37,3 \mathrm{IFC} \%)$ y la categoría visión (7,1 IFC\%). En sintonía, se podría argumentar que la intención (visión) de desarrollar un liderazgo pedagógico se diluye en el camino hacia su puesta en práctica en donde los requerimientos y, sobre todo, las resistencias, centran las claves del discurso.

Ahora bien ¿qué tipo de resistencias y/o limitaciones se manifiestan en pos de materializar el liderazgo pedagógico? La organización de los indicadores establece el siguiente orden -decreciente- traducido en siete sub-categorías: a) la formación pedagógica del profesorado (15,3 IFC\%); b) la situación jerárquica - grado de autoridad (12,8 IFC\%); c) el modelo de dirección (10,3 IFC\%); d) la gestión del profesorado (6,3 IFC\%); e) la formación específica para el cargo (5,3 IFC\%); la realización de tareas burocráticas (2,8 IFC\%); [y en menor medida] el grado de autonomía (2,8 IFC\%).

Gráfico 1 - IFC porcentual de categorías y sub-categorías emergentes.

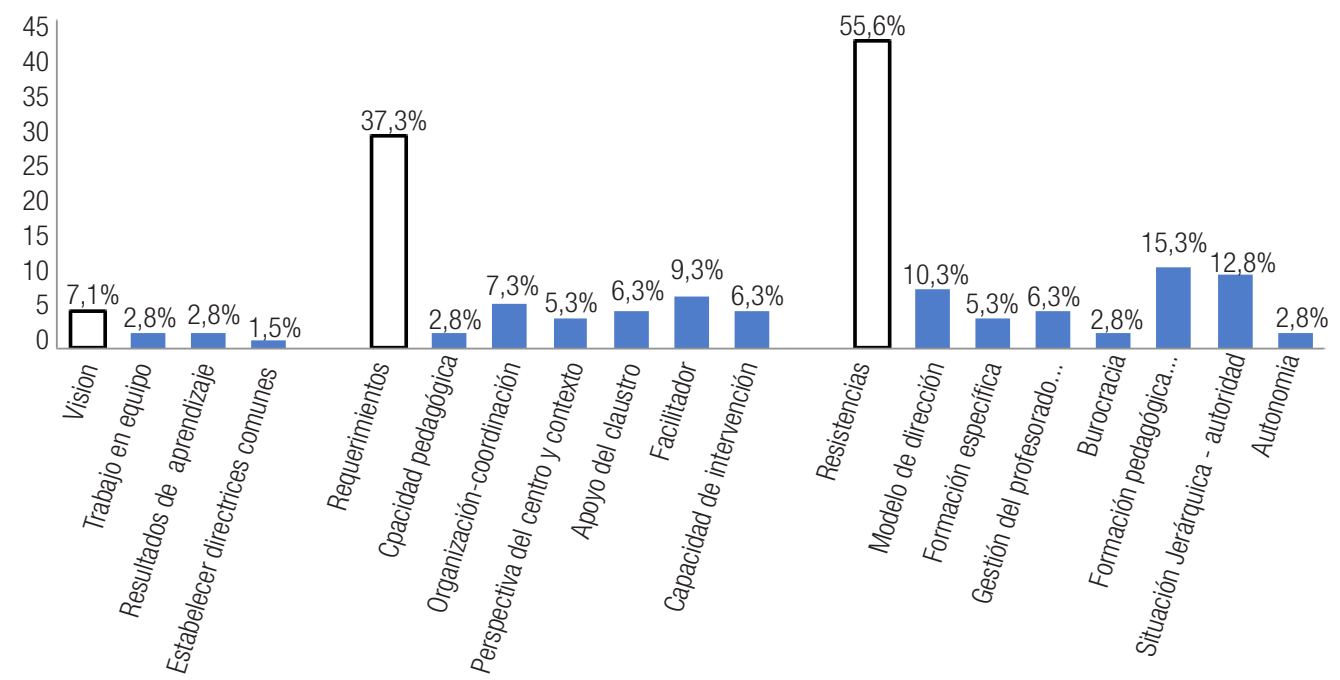

Fuente: Elaborado por los autores.

Desde un enfoque más analítico, se observan diferentes niveles de resistencias. El telón de fondo, lo configura el modelo español de dirección escolar (itinerante-transeúnte) que atañe a la compleja situación jerárquica de los directores (intermedia, entre la Administración y el cuerpo docente). En este sentido, refieren a su escaso grado de autoridad y autonomía pedagógica. A su vez, como una de las consecuencias del diseño corporativo para la toma de decisiones (dependencia del claustro y del consejo escolar), es posible justificar gran parte de las argumentaciones que hacen referencia a -centrar su actividad en tareas burocráticas-. 
En sintonía, la escasa formación específica para ejercer el cargo y la falta de formación pedagógica del profesorado se sitúan como aspectos agravantes.

La categoría requerimientos configura una estructura ciertamente pareja en la distribución de sus indicadores. En este marco, se establecen una serie de continuidades con la categoría anterior. Es decir, ante la limitación en la capacidad de autoridad y autonomía, emergen la necesidad el apoyo del claustro (6,3 IFC\%) y de intervención (6,3 IFC\%). Así pues, el margen de maniobra que le permite su dedicación a tareas burocráticas, si bien no le posibilita desarrollar su capacidad pedagógica (2,8 IFC\%) en los procesos de enseñanza-aprendizaje del aula (diagnóstico, asesoramiento, evaluación), le refuerzan como un organizador- coordinador (7,3 IFC\%) que facilita (9,3 IFC\%) la tarea del personal en pos de un buen clima de trabajo en el centro educativo. Para ello, se considera clave, en términos de capital social, que sea capaz de integrar una perspectiva micro-politica de su centro y del contexto educativo (5,3 IFC\%) en el que se encuentra.

En términos de IFC\%, la categoría visión no solo se refleja como la menos referenciada, sino también, como la que presenta el menor número de sub-categorías. No obstante, merece la pena prestar atención a aquellos aspectos que evidencian expresiones de deseo por un liderazgo pedagógico. Conectado con elementos de las categorías anteriores (autoridad, coordinación, facilitador, etc.), se valora potenciar el trabajo en equipo (2,8 IFC\%) y establecer directrices comunes (1,5 IFC\%) como formas de mejorar los resultados de aprendizaje (2,8 IFC\%) del alumnado.

En otro orden, el Gráfico 2 representa la distribución de indicadores (I) en función del ISEC de los centros educativos. Al respecto, es posible observar cómo los centros de ISEC bajo concentran un 46\% de total de las unidades de análisis (UA) identificadas. Seguidos, de forma casi paralela, los centros de ISEC medio, con un 25\%, se equiparan a los de ISEC alto (29\%). Este aspecto podría estar dando cuenta de la incidencia del factor socio-económico y cultural en los centros educativos. En esta línea, parece denotarse el incremento del interés por el desarrollo del liderazgo pedagógico cuando el contexto escolar presenta un mayor reto y dificultades para el logro del desarrollo educativo del alumnado (MURILLO, 2003).

Gráfico 2. IFC porcentual indicadores por tipo de centro (ISEC).

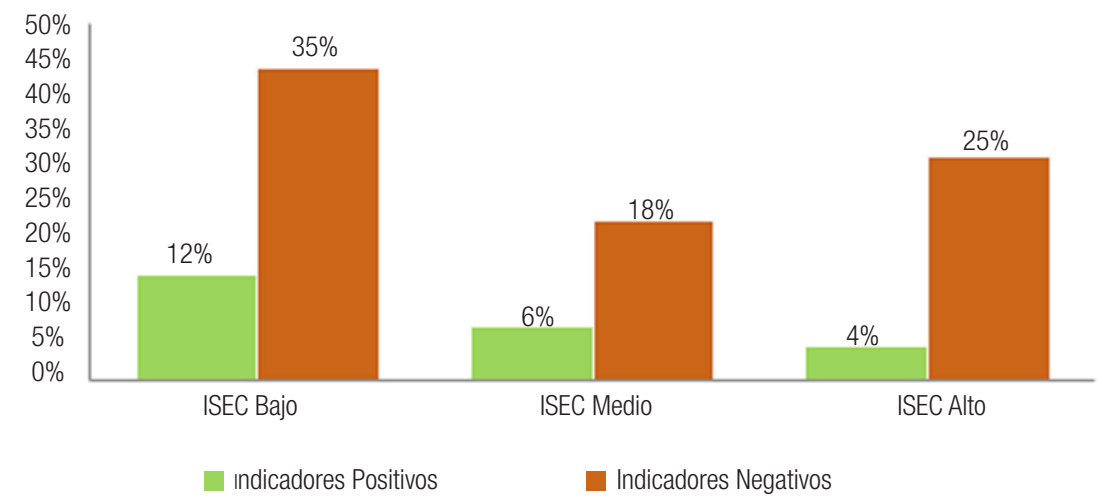

Fuente: Elaborado por los autores. 
El Gráfico 3 avanza en el sentido de la distribución de las unidades de análisis (UA) del Gráfico 2. El cruce entre el ISEC y el tenor positivo o negativo del contenido de las UA manifiesta cómo -las referencias negativas con relación al desarrollo del liderazgo pedagógico superan, en los tres tipos de centro, a las positivas-. Esta tendencia es mayor en los de ISEC bajo (diferencial de 23 puntos porcentuales) así como las referencias negativas (35\%). A colación, reiteramos la interpretación de la -incidencia del factor situacional en el ejercicio del liderazgo pedagógico en zonas desfavorecidas. No obstante, es en estos centros donde se registra el mayor porcentaje de referencias positivas (12\%) clarificando la idea de que -un mayor grado adversidad también es susceptible de activar dinámicas que conlleven a un liderazgo pedagógico para la mejora-.

Gráfico 3 - IFC porcentual indicadores positivos y negativos por tipo de centro (ISEC).

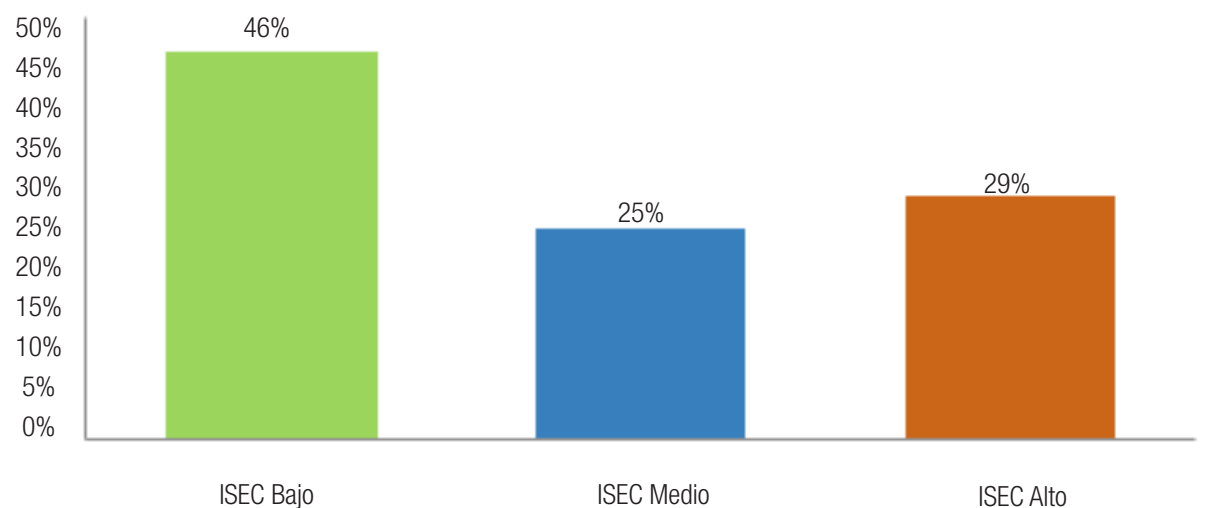

Fuente: Elaborado por los autores.

El gráfico 4 refleja el cruce entre el las categorías emergentes y el tipo de centro. Se destaca, en los tres contextos, el incremento del IFC en el sentido visión-requerimientosresistencias. Se subraya un aspecto ya abordado en los Gráficos 2 y 3 -los de ISEC bajo, al tiempo que encabezan la categoría resistencias (referencias negativas) también lo hacen en la categoría visión (referencias positivas) y requerimientos.

Se expresa significativa la distribución de los indicadores en la categoría requerimientos. En este marco, los centros de ISEC bajo igualan su IFC (20\%) con la categoría resistencias. Esta cuestión reafirma la idea de que en contextos desfavorecidos los directivos se muestran activos ante las contingencias a superar, en otras palabras, -son conscientes de las dificultades (resistencias), y en igual medida, reclaman aquellos elementos que pueden ejercer un cambio hacia un liderazgo de corte pedagógico-.

Por otro lado, se considera la paridad del IFC porcentual de los tres tipos de centro en la categoría resistencias, dando cuenta que las dificultades para desarrollar un liderazgo pedagógico inciden, más allá de las características socio-económicas y culturales (MORAL; AMORES, 2014). 
Gráfico 4 - IFC\% Categorías emergentes cruce con ISEC de centros educativos.

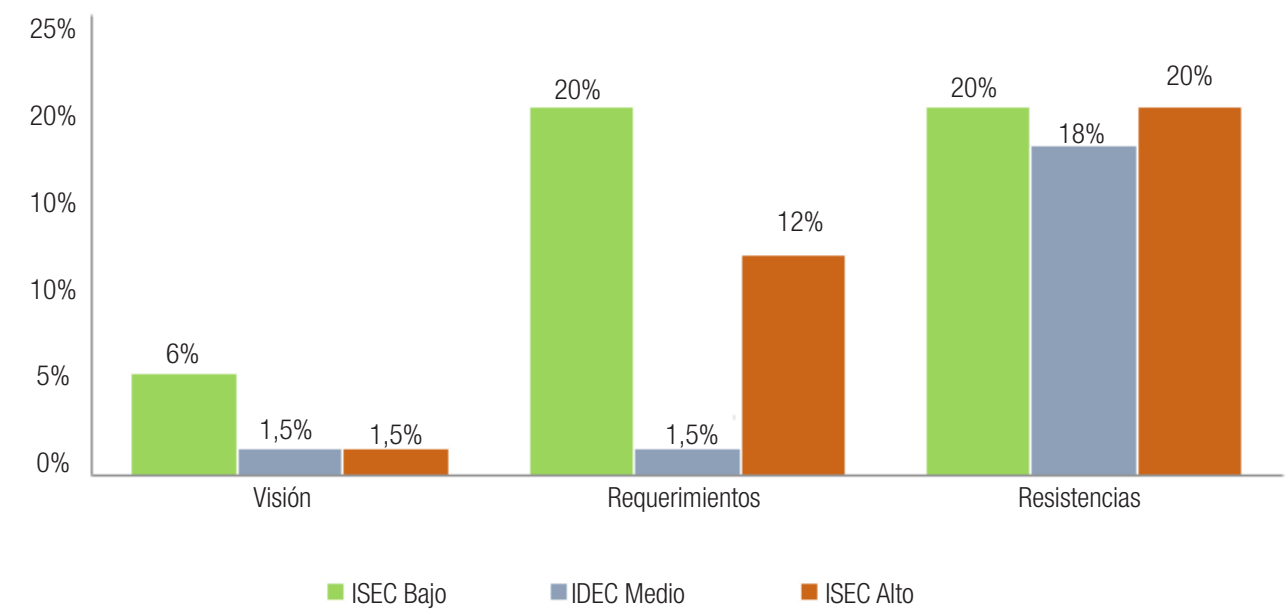

Fuente: Elaborado por los autores.

Sin más, en el próximo apartado se expondrá la teoría fundamentada que refleja el sentido interpretativo de los resultados del estudio. En este marco, nos centramos en el valor empírico de los fragmentos identificados en las entrevistas y en el enfoque testimonial del corte biográfico-narrativo (CHARMAZ, 2004).

Segundo y tercer nivel de profundización. Del análisis de contenido a la teoría fundamentada

Una aproximación más profunda al análisis de los indicadores categoriales identifica elementos del discurso que merecen ser tenidos en cuenta. En sintonía, por medio del testimonio de los entrevistados, se ponen de manifiesto las valoraciones respecto al ejercicio del liderazgo escolar y el desarrollo del liderazgo pedagógico, cómo son percibidas estas cuestiones en el marco de la función directiva.

Visión de un liderazgo pedagógico

La categoría visión refiere a la perspectiva de los directores acerca del significado del liderazgo pedagógico. El término en sí abarca el desarrollo de una serie de capacidades (SPILLANE, 2006; DAY; GU; SAMMONS, 2016), no obstante, bajo la mirada situacional de los entrevistados, establecer directrices comunes y el trabajo en equipo son aspectos prioritarios:

Tener un liderazgo pedagógico es básico porque tienes que dirigir a compañeros [...] comienza con los propios miembros del equipo directivo...no es uno solo...es el claustro con los que uno cuenta para para dirigir organizar o gestionar el centro $(\mathrm{C} 12)^{5}[\ldots]$ Liderar es establecer el "hacia

5- La codificación de los centros responde a la siguiente nomenclatura de codificación: C1 al C5 (Centros de ISEC Alto); C6 al C10 (Centros de ISEC Medio); C11 al C15 (Centros de ISEC Bajo). 
dónde vamos" $(\mathrm{C} 11)^{6}[\ldots]$ se trata de transformar el trabajo individual del profesorado en ir avanzando hacia un trabajo en equipo(C1).

Se denota que el acento por desarrollar el trabajo en equipo responde a la necesidad de apoyo para liderar la organización. Ser parte de un modelo corporativo seguramente incentiva estos procesos (BOLÍVAR, 2012; 2014a). Por otra parte, en el avance hacia un modelo post-burocrático se comienza a difuminar aquella imagen heroica de los directores de antaño (MORAL; AMORES; RITACCO, 2016). En este orden, también se resaltó la capacidad para obtener resultados:

La función principal es trasformar, ir haciendo ajustes y ejerciendo iniciativa para que todo funcione mejor y para que los alumnos aprendan y vayan sacando mejores resultados (C2) [contrastando con uno de los centros de ISEC bajo] el liderazgo pedagógico no solamente son los resultados académicos, son las necesidades del centro y de un alumnado con muchas dificultades socioeducativas (C11).

Acorde con Day, Gu y Sammons (2016) el enfoque del liderazgo pedagógico debe implicar, indefectiblemente, la mejora de los resultados de aprendizaje del alumnado. Sin embargo, como se expresa en los fragmentos, la realidad de los contextos más desfavorecidos fuerza a rebajar las expectativas hacia un liderazgo que responda a aspectos educativos más básicos (habilidades sociales, tasa de asistencia, etc.).

\section{Requerimientos para un liderazgo pedagógico}

Avanzando en la construcción interpretativa de los resultados, la categoría requerimientos abarca un conjunto de capacidades que son consideradas necesarias para alcanzar un liderazgo escolar a la altura de los tiempos que se están viviendo. Entre las más referenciadas se comenta que el director sea un facilitador:

[En lo pedagógico] Debo ser un facilitador para el profesorado, estimular la renovación metodológica y pedagógica, proponer más práctica, nuevas herramientas, facilitarle el trabajo (C12) [A nivel organizativo] Quiero ser un facilitador, un creador de condiciones organizativas en el centro para que la gente se sienta cómoda para innovar, mejorar, formarse. (C11) [Media $y$ orienta al personal] un director debería cubrir un cierto papel de moderador, de guía [...] uno debe sencillamente orientar para que la gente se implique. (C7) [Facilita un clima positivo de trabajo] quisiera ser más un facilitador, preocuparme por ofrecer buenos proyectos educativos y generar un buen ambiente de relación personal. (C3).

Acorde con lo anterior, se destaca la capacidad coordinativa del director, que sea un buen coordinador. A priori, se clarifican una serie de aspectos que pueden hacer más efectiva esa tarea:

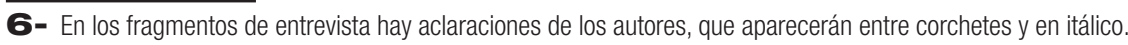


[Lineas de actuación] El liderazgo pedagógico se facilita con una buena coordinación que tenga una líneas pedagógicas muy claras (C1) [...] Para liderar hay que establecer unas líneas que se apoyen en una buena coordinación (C4) [Estructura de coordinación] no se puede liderar desde la perspectiva individual, hay que hacerlo de forma coordinada [...] el trabajo debe ser coordinado departamentalmente para que el profesorado tenga unas pautas pedagógicas comunes [...] luego los departamentos también tienen que funcionar coordinados en el marco del proyecto educativo de centro liderado por el equipo directivo (C3) [Gestión de recursos] hay que coordinar sabiendo arbitrar los recursos [...] para coordinar debemos hacer malabares con los recursos, eso es lo que hicimos desde un principio.(C11).

Otra cuestión que emerge con fuerza es el tema de la capacidad de intervención de la dirección. De los indicadores identificados se resalta el tenor negativo del discurso asociado a una serie de elementos que dificultan el desarrollo de esta capacidad:

[Naturaleza de las tareas-administrativas] El problema es que estoy aislado, todo el día encerrado con proyectos, mandando, revisando, etc., y no me permite intervenir personalmente. (C15) [...] para liderar se te tiene que ver interviniendo personalmente y así das ejemplo con tu labor, pero eso cuesta mucho ya que estoy atado al ordenador, a lo administrativo y no se me ve. (C12) [...] Somos excesivamente gestores y hay demasiada carga administrativa y eso te priva de tener contacto con los compañeros. (C13) [Naturaleza de las tareas-resolución de problemas] Soy el que va apagando los fuegos cotidianos del personal [...] ese es el tipo de intervención que realiza un director. (C15).

Como se manifiesta, estas situaciones atomizan la función de la dirección, limitan su campo de actuación pedagógica. En este orden, el margen de maniobra se reduce aún más si tenemos en cuenta el peso que se le confiere al apoyo del claustro (modelo corporativo):

El claustro te tiene que respaldar y apoyar, si no tienes ese apoyo mejor no te presentes a director [...] es que tienes que tener ese apoyo. (C12) [...] Para construir un liderazgo necesitas el apoyo del claustro, que crean en ti, que vean que tienes posibilidades que tienes capacidad para dirigir el centro. (C8).

Por ello, en este marco, quien pretenda ejercer la dirección debe tener una radiografía clara del capital social y micro-político de su centro, una perspectiva ecológica-global que clarifique los núcleos de poder e influencia como punto de partida al establecimiento de estrategias pedagógicas, políticas organizativas, etc.:

Una de las labores del director, como ese líder que debe de ser, es observar por donde circulan las líneas de poder entre las relaciones del profesorado y desde ahí desarrollar tu proyecto. (C4) [...] Es central saber ver por dónde van los flujos de poder entre las personas, las luchas digamos [...] hay líderes en los claustros, el director no es el único líder, hay que contar con ellos ya que con ellos te ganas a mucha gente que está detrás.(C2) [Con una perspectiva más amplia del centro] Hay que construir una radiografía del centro, las necesidades del alumnado, que profesorado tienes, los 
recursos que cuentas.(C12) [...del contexto y la comunidad educativa] Saber dónde está tu centro, la comarca, la zona, los datos, conocer la situación, luego debes marcarte tu norte y objetivos. (C11).

Se dilucida el peso significativo y la importancia que adquiere este tipo de habilidades para la puesta en práctica y desarrollo de un liderazgo con capacidad pedagógica (BOLÍVAR, 2012). No obstante, en nuestro estudio, las referencias de la sub-categoría capacidad pedagógica no llegan a alcanzar un nivel de práctica capaz de responder al desarrollo pleno de un liderazgo pedagógico:

El director debe de ser el líder pedagógico del centro y eso se refleja en nuestro proyecto de dirección [...] consideramos que para solucionar temas como el absentismo, convivencia, etc., la forma de enfrentarlo es mediante lo pedagógico [...] llevarlo a la práctica es lo más complicado, estoy muy limitado a la hora de asesorar a mi profesorado (C15) [...] En mi centro lo pedagógico es al mismo tiempo un eje y lo más difícil de llevar a cabo [...] no tengo autoridad pedagógica en la práctica, solo en la teoría, y a regañadientes. (C12).

Como resaltamos en pasadas investigaciones (RITACCO; AMORES; MORAL, 2015; Moral; AMORES; RITACCO, 2016), en España, la cultura escolar dificulta el avance del asesoramiento pedagógico de la dirección, en consecuencia, las actuaciones quedan en un plano de elaboración, iniciativa, propuesta, lejos de producir cambios en la práctica del aula.

\section{Resistencias para el desarrollo del liderazgo pedagógico}

La fase interpretativa del análisis de los resultados se cierra con la categoría resistencias. La comprensión emergente del discurso ubica a la sub-categoría modelo de dirección en la base de la teoría fundamentada. En sintonía, en los tres tipos de centro se coincide en resaltar aspectos del modelo de dirección que están impidiendo el desarrollo del liderazgo pedagógico:

[Temporalidad en el cargo - itinerario transeúnte - limita la toma de decisiones] hay circunstancias que impiden los avances y una de ellas es que el puesto de director no es permanente [...] a uno se le asciende y se le degrada dentro del mismo centro. (C9) [...] estamos de paso en la dirección, vamos a cuatro años y volvemos a ser docentes, entonces ise pueden avanzar considerando esta limitación? (C1) [Sistema abierto de elección - corporativo - posicionamiento inter-niveles] Nos elige el claustro, es decir, la comunidad educativa, y la administración, eso nos mediatiza mucho [...] es complicado y complejo a la vez. (C12) [...] Apenas te nombran te cargan de competencias que son de gestión y de representación de la administración [...] un representante del profesorado ¿Qué atiende los intereses del profesorado, de las familias, del alumnado? que va... [...] estoy a la deriva entre los intereses de todos [...] si yo soy director será porque el profesorado me ha votado ¿no? pero de cara a ellos yo represento a la administración. (C3) [Impacto en las dinámicas] Me limito a sacarle oportunidades a la consejería de educación y luego ofrecerlas a mi profesorado, a las familias. (C3) [...] debo ser capaz de utilizar lo que me ofrece la administración, ver lo que me puede 
servir [...] luego concretarlo y contextualizarlo para que el profesorado lo vea con buena cara y trabaje alrededor de eso. (C8).

A la luz del contenido de los fragmentos queda expuesto que el propio sistema de elección, de base, refuerza el posicionamiento (inter-niveles) de la dirección produciendo un impacto en su práctica diaria. Así pues, toma cuerpo la idea de vulnerabilidad en el intento de ejercer las funciones directivas (RITACCO; AMORES; MORAL, 2015). Esta cuestión podría justificar que se manifieste el reclamo de profesionalizar la carrera directiva:

[Estatus para el cargo] Hasta que la dirección no esté concebida en España como carrera profesional, será simplemente un cargo sin identidad. (C4) [...] cuando llegas te das cuenta que eres un gestor administrativo y que además no tienes formación para ello! (C5) [...]Yo casi fui director al llegar a mi primer centro, me nombraron vice, iy acababa de aprobar las oposiciones! ¡Sin formación ninguna! ¿iCómo eso podía ser posible!? (C13) [Hacia la profesionalización] te encierras en una gestión administrativa, te absorben las demandas del claustro y de la administración y muy poco de ese liderazgo pedagógico [...] esto se debe a una falta de formación específica. (C2) [con un itinerario de formación especifico] la dirección debería ser una carrera profesional diferenciada, así como la inspección educativa.(C1) [...] somos directores sin ni siquiera una carrera que nos acredite como tal. (C8).

En este orden, el tema de la formación pedagógica no deja exento al profesorado. Se manifiesta la necesidad de mejorar las competencias pedagógicas de los profesionales de la educación:

[La falta formación pedagógica] La principal dificultad que hay en secundaria es que la propia formación pedagógica del profesorado es bastante escasa. (C12) [...] en general tenemos licenciados, especialistas en sus áreas, con muy poca formación pedagógica. (C14) [...] A la enseñanza llega mucha gente sin formación pedagógica (C3) [Reciclaje profesional] la profesión docente no integra itinerarios obligatorios de renovación pedagógica. (C7) [...] el profesorado puede perfectamente sacar sus oposiciones y no hacer cursos de formación a lo largo de toda su vida docente. (C10) [Reticencia al asesoramiento pedagógico] al profesorado, pedagógicamente, no se le puede decir lo que tiene que hacer. (C3) [...] es muy complicado sugerirle a un profesor algo en referencia a sus sesiones en el aula, eso les pone muy mal, y al final, no consigues nada, consigues enfrentamientos. (C5).

Ante este panorama se abre el debate acerca de la potestad real de los directores escolares para incentivar o conducir al personal hacia la mejora del ejercicio pedagógico de sus funciones. El grado de autoridad asociado a su estatus jerárquico en la estructura formal debería ayudar a encaminar este aspecto, sin embargo, se identifican una serie de limitaciones:

[Toma de decisiones] Es muy difícil liderar y marcar una forma de trabajar cuando realmente tengo muy poca capacidad de decisión sobre profesorado. (C3) [...] si no tengo capacidad para 
tomar decisiones ¿cómo voy a marcar, dirigir y liderar un proyecto pedagógico real? (C2) [...] [Autoridad entre iguales] es difícil dado que el director es un compañero docente y el respeto y la autoridad por eso es relativa (C14) [...] Lo primero que impide el buen ejercicio del liderazgo es esa relación que tenemos con nuestros compañeros y los seguimos llamando -compañeros- es muy curioso pero yo sigo siendo "compañera de..." claro iy soy la directora!(C3) [...] aquí todos somos compañeros y esa idea es rara [...] ellos en el fondo lo entienden así y no te ven como alguien al que hay que cumplirle las cosas. (C8) [...] [Necesidad de habilidades -no formales] te los tienes que ganar con el ejemplo, demostrando que tienes cierta formación. (C14) [...] Tenemos que tener más habilidades humanas que ejecutoras [...] con habilidades humanas vamos convenciendo a la gente y eso es lo complejo, el tener que establecer más relaciones humanas. (C7) [Necesita apoyos] aquí la autoridad se logra con el respaldo de la comunidad educativa. (C9) [...] el liderazgo pedagógico no se puede ejercer cuando en el claustro existen sectores que no comparten tu planteamiento [...] ellos pueden perfectamente poner en duda tu capacidad como director. (C10) [...] la principal reticencia proviene mis propios recursos humanos [...] si no tengo el apoyo del claustro difícilmente podré desarrollar mi proyecto educativo. (C8).

Ampliando un poco la perspectiva, se denota cómo la responsabilidad de los directores por ejercer autoridad contrasta con los aspectos que dificultan el proceso de toma de decisiones. En cualquier caso, la tensión que generan estas situaciones se centran en la dirección. Un ejemplo claro se produce a la hora de gestionar al profesorado luego de un cambio normativo que incrementaba la carga horaria del profesorado:

Es muy difícil desarrollar el liderazgo pedagógico cuando la administración le sube a veinte las horas lectivas al profesorado. (C13) [...] La subida a 20 horas del profesorado inunca creí que me iba a afectar tanto! [...] no puedo componer mi equipo con esta subida, no me dan juego, no cuento con unas horas de funcionamiento y no me cuadra el horario [...] voy a estar a expensas de la disponibilidad de horario de los departamentos. (C15) [...] Con el aumento de horas ya llegamos a una situación límite aquí ya todos somos plantilla orgánica y tú no tienes posibilidades y margen de maniobra para componer un buen equipo pedagógico. (C11) [...] las limitaciones son impuestas y por esta subida de horarios, esas dos horas, nos ha llevado a una situación sin margen de gestión pedagógica. (C12).

Las circunstancias descritas reducen claramente la capacidad de ejercer el liderazgo pedagógico. En relación, el grado de autonomía de la dirección, muy citado en la LOE, Ley Orgánica de Educación (ESPAÑA, 2006), pierde fuerza cuando los niveles superiores aumentan el control -gobernabilidad- de los centros:

La propia administración nos llena de tantas tareas administrativas que te puedo decir yo que lo de la autonomía es una falacia (C6) [...] En la gestión, es tanto el papeleo que no nos da tiempo para nada, nos quita autonomía (C4).

Paralelamente, también se incrementa uno de los mecanismos de regulación, el requerimiento de información, generando un gran cúmulo de tareas administrativas -burocracia-: 
El liderazgo pedagógico exige una dedicación muy específica y desgraciadamente no hay disponibilidad de horas para hacerlo y tenemos que preparar y dar clases, y hay mucha burocracia que te limita. (C13) [...] Una gran traba es el exceso de burocracia, para cualquier cosa son montones de pilas de papeles que hacer, se pierde mucho tiempo y eso dificulta mucho. (C8).

\section{Conclusiones}

La investigación sobre successful school principals ha estado dominada por la indagación acerca de qué tipo de prácticas tienen un impacto en los aprendizajes de los estudiantes y en los resultados de la escuela. Dentro de este marco, una mentalidad en exceso performativa, centrada en competencias técnicas, nos ha hecho silenciar la perspectiva cualitativa de las investigaciones. En efecto, se ha podido relegar el alcance de un enfoque hermenéutico capaz indagar en el mundo de la vida de los directores e interpretar sus relatos. En este caso, la dimensión testimonial y biográfica ocupa un lugar central (MERRIAM, 2009). Siendo relevante esta faceta, somos tácitos en afirmar que la otra cara del liderazgo, la de la relación e interacción con los demás, no es un fenómeno periférico, sino que constituye un elemento central al momento de entender los factores que inciden en el ejercicio del liderazgo pedagógico (CROW; DAY; MÖLLER, 2016).

Bajo este prisma, consideramos, entre tantas, dos ideas clave para comenzar a profundizar en lo que está sucediendo con el liderazgo escolar en España. En primer lugar, nos afirmamos en la paradoja de que poseemos escuelas fuertemente uniformadas, sin protagonismo de su dirección y desvertebradas por la inercia de la tradición burocrática (BOLÍVAR, 2012). En segundo, de un modo más explicativo, el modelo actual de dirección escolar está sujeto a un amplio abanico de factores mediados por una fuerte cultura escolar.

De un modo transversal, dichas cuestiones están presentes en el estudio, impregnan los resultados obtenidos, y se observan en las categorias emergentes que hacen referencia al ejercicio del liderazgo en función del desarrollo de un liderazgo pedagógico. Bajo este enfoque, visión, requerimientos y resistencias son, en gran medida, expresiones por reconvertir el liderazgo a un modo más pedagógico. El peso de la información da cuenta de ello, incluso, de cómo se incrementa la preocupación y la inquietud por estas cuestiones cuando se presenta adverso el factor socio-económico y cultural del centro.

Emergente del análisis de contenido, el contínuum interpretativo de la teoría fundamentada establece que la visión de los entrevistados refiere al significado del liderazgo pedagógico. Nada en desacuerdo con la bibliografía recomendada, se sobrevalora el establecer directrices comunes y el trabajo en equipo (PERRY, 2009; SPILLANE, 2006; BOLIVAR, 2012; DAY; GU; SAMMONS, 2016). Lo significativo de ambas cuestiones se apoya en dos aspectos clave. Por un lado, la necesidad de contar con colectivo profesional para liderar la organización, y por otro, que el logro de estas dinámicas radica en arduos procesos de negociación que requieren de las habilidades sociales por parte del líder escolar (MORAL; AMORES; RITACCO, 2016).

Con el análisis de la categoría requerimientos se avanzó en la comprensión de la situación. El criterio que conformó el contenido categorial se definió por aquellas 
capacidades que posibilitarían alcanzar un liderazgo pedagógico. Al respecto, facilitar y organizar-coordinar, si bien centrales, juegan en la periferia cuando hablamos de una capacidad pedagógica real que pueda producir cambios en el aula. Exento de la posibilidad de avanzar en lo pedagógico, se reduce el vértice de actuación e intervención hacia la realización de tareas administrativas (burocráticas). En este sentido, para la toma de decisiones, el apoyo del claustro está sobrevalorado y reforzado por un sistema básicamente corporativo. En este punto, cotiza en alza un conocimiento general y social del centro, una perspectiva ecológica del terreno que permita futuras y fructíferas negociaciones con el personal para el desarrollo de las líneas de trabajo.

Las resistencias terminan de clarificar el panorama. Es que si reflexionamos acerca del enfoque del liderazgo pedagógico, el modelo de dirección en España hace aguas en su diseño (MURILLO, 2003; BOLÍVAR et al., 2004; DOMINGO; RITACCO, 2015). El itinerario de ida y vuelta en el cargo (transeúnte), la falta de una formación específica y el sistema de elección (corporativo), referenciados en este estudio, ubican a los directores en una situación cautiva entre las demandas de la administración y la comunidad educativa. Entre la confusión y la complejidad se suma la circunstancia de ser, en una dimensión paralela, un director-compañero docente. Es de esperarse pues, que en la práctica, mientras se incrementa el condicionamiento a la hora de tomar decisiones se reduzca la posibilidad de poner en valor su autonomía y su autoridad formal. En este punto, para ganar apoyos (y capital social), entran en juego las ya mencionadas habilidades sociales necesarias para, por ejemplo, incentivar la formación pedagógica del profesorado.

Desde una perspectiva más amplia, el modelo español de dirección escolar merece ser analizado desde la complejidad del rol docente-director-docente, considerando las tensiones que genera ocupar un posicionamiento intermedio entre las demandas de la administración y las negociaciones con sus colegas del profesorado. Esta encrucijada se agrava si se tiene en cuenta el itinerario de ida y vuelta -transeúnte- por el cargo y su sistema de elección, ciertamente corporativo.

Por otro lado, considerando los testimonios de los entrevistados, no es de extrañar que se esté precisando una capacitación específica (formación inicial y en servicio), en particular, habilidades y estrategias para hacer del centro una comunidad centrada colectivamente en la mejora de la educación ofrecida. Como bien afirmaba Bolívar (2012), el estado de la cuestión no debate en si es más o menos formación, sino en que marco y/o coordenadas se inscribe.

Sin más, del estudio emanan una serie de líneas de investigación. En nuestro caso, en estos últimos meses hemos avanzado en el impacto del modelo español de dirección escolar en las diferentes dimensiones de la identidad de los directores escolares (RITACCO; BOLÍVAR, 2016). En este sentido, a lo largo de su trayectoria profesional, duplicidades y discontinuidades (un docente que llega a la dirección para retornar en el futuro al cuerpo docente) exponen a los directores de los centros educativos a una situación identitaria que lo aleja de alcanzar, entre otros aspectos, un liderazgo pedagógico para la mejora del aprendizaje del alumnado.

Por ello, abriendo la perspectiva, si pretendemos orientar nuestros esfuerzos hacia la mejora escolar por medio de un liderazgo transformacional-pedagógico, será 
menester revisar un mínimo de líneas para el cambio. Entre otras, emanadas del estudio, nos permitimos proponer: dotar a los directores de una capacidad real para intervenir en los procesos pedagógicos; que la dirección escolar se reconvierta en una profesión atractiva en el sentido más amplio del concepto de autonomía, sin perder esa visión colectiva y colaborativa del centro; pasar de un modelo corporativo (colegas que eligen) a uno transformacional que permita una toma de decisiones sin sesgos ni compromisos corporativos.

\section{Referencias}

ÁVILA DE LIMA, Jorge. Por uma Análise de conteúdo mais fiável. Revista Portuguesa de Pedagogia, Coimbra, v. 47, n. 1, p. 7-29, 2013.

BARBER, Michael; MOURSHED, Mona. Cómo hicieron los sistemas educativos con mejor desempeño para alcanzar sus objetivos. Santiago de Chile: Mckinsey and Company, 2007.

BARROSO, João (Org.). A regulação das políticas públicas de educação: espaços, dinámicas e actores. Lisboa: Educa, 2006.

BOLAM, Ray et al. (Org.). Creating and sustaining effective professional learning communities. Bristol: University of Bristol, 2005. Research report RR637.

BOLIVAR, Antonio. ¿Cómo un liderazgo pedagógico y distribuido mejora los logros académicos? Revisión de la investigación y propuesta. Magis, Bogotá, v. 3, n. 5, p. 34-54, 2010.

BOLIVAR, Antonio. Construir la capacidad de mejora escolar: liderazgo compartido y comunidades profesionales de aprendizaje. International Journal of Educational Leadership and Management, v. 2, n. 2 , p. 23-54, 2014a.

BOLIVAR, Antonio et al. Evaluación de la función directiva en centros sostenidos con fondos públicos. Enseñanza, Salamanca, v. 22, p. 35-76, 2004.

BOLIVAR, Antonio. Las historias de vida del profesorado: voces y contextos. Revista Mexicana de Investigación Educativa, México, DF, v. 19, n. 62, p. 711-734, 2014 b.

BOLIVAR, Antonio. Políticas actuales de mejora y liderazgo educativo. Málaga: Aljibe, 2012.

BROOKS, Jeffrey; NORMORE, Anthony. Qualitative research and educational leadership. International Journal of Educational Management, n. 29, p.798-806, 2015.

CHARMAZ, Kathy. Grounded theory: objectivist and constructivist methods. In: Emerson, Robert (Ed.). Contemporary field research. Boston: Little, Brown and Company, 2004. p.109-126. 
COMISIÓN EUROPEA. Mejorar las competencias en el siglo XXI: agenda para la cooperación europea en las escuelas. Bruselas: Comisión de Comunidades Europeas, 2008. Comunicación de la Comisión al Parlamento Europeo, al Consejo, al Comité Económico y Social Europeo y al Comité de las Regiones.

COMISIÓN EUROPEA. Progress toward the Lisbon objectives in education and training: analysis of implementation at the European and national levels. Bruselas: Comisión de Comunidades Europeas, 2009.

CROW, Gary; DAY, Cristopher; MÖLLER, Jorunn. Framing research on school principals' identities. International Journal of Leadership in Education, v. 20, n. 3, p. 265-267, 2016. Disponible en: <http:// dx.doi.org/10.1080/13603124.2015.1123299>. Acceso: 26 feb. 2016.

DAY, Christopher; GU, Qing; SAMMONS, Pam. The impact of leadership on student outcomes: how successful school leaders use transformational and instructional strategies to make a difference. Educational Administration Quarterly, v. 52, n.2, p. 221-258, 2016.

DOMINGO, Jesús; RITACCO, Maximiliano. Aporte del departamento de orientación al desarrollo del liderazgo pedagógico: un estudio desde la opinión de directores de institutos de enseñanza secundaria en Andalucía. Educar em Revista, Curitiba, n. 58, p. 199-218, 2015.

ELMORE, Richard. Mejorando la escuela desde la sala de clases. Santiago de Chile: Fundación Chile, 2010.

ESPAÑA. Ministerio de Educación Cultura y Deporte. LOE - Ley Orgánica de Educación. B0E, Madrid, n. 106, p. 17158-17207, 4 mayo 2006. Disponible en: <https://www.boe.es/boe/dias/2006/05/04/pdfs/A1715817207.pdf>. Acceso: 26 feb. 2016.

FERNANDA, Viviani; ZAMBÃO, Graziela. Perfil do diretor da escola pública estadual paulista. Educação, Santa Maria, v. 40, n. 1, p. 201-214, 2015.

FLICK, Uwe. Introducción a la investigación cualitativa. Madrid: Morata, 2007.

FULLAN, Michael. Las fuerzas del cambio. Explorando las profundidades de la reforma educativa. Madrid: Akal, 2002.

GIMENO, José et al. La dirección de centros: análisis de tareas. Madrid: CIDE-MEC,1995.

GUBA, Egon; LINCOLN, Yvonna. Competing paradigms in qualitative research. In: DENZIN, Klaus; LINCOLN, Yvonna (Ed.). Handbook of qualitative research. London: Sage, 1994. p. 105-117.

HARGREAVES, Andrew; FULLAN, Michael. Capital profesional. Madrid: Morata, 2014.

KOETTING, John. Foundations of naturalistic inquiry: developing a theory base for understanding individual interpretations of reality. Dallas: Association for Educational Communications and Technology, 1984.

KRIPPENDORFF, Klaus. Metodología del análisis de contenido: teoría y práctica. Barcelona: Paidós Ibérica, 1990. 
LÓPEZ-YAÑEZ, Julián; LAVIÉ, José. Liderazgo para sostener procesos de innovación en la escuela. Profesorado, Granada, v. 14, n. 1, p. 71-92, 2010.

MAROY, Christian. Convergences and hybridación of educational policies around 'postbureaucratic' models of regulation. Compare, v. 39, n. 1, p. 71-84, 2009.

MASON, Pamela. Liderança escolar: desenvolvimentos de comunidades de prática. Revista Pesquisa e Debate em Educação, Juiz de Fora, v. 1, n. 1, p. 59-66, 2011.

MAYNTZ, Renate. El estado y la sociedad civil en la gobernanza moderna. Revista del CLAD, n. 21, p. 1-8, out. 2001.

MERRIAM, Sharan. Qualitative research: a guide to design and implementation. San Francisco: JosseyBass, 2009

MORAL, Cristina; AMORES, Francisco. Arquitectura resistente determinante del liderazgo pedagógico en los centros de Secundaria. Bordón, v. 66, n. 2, p. 121-138, 2014.

MORAL, Cristina; AMORES, Francisco; RITACCO, Maximiliano. Liderazgo distribuido y capacidad de mejora en centros de educación secundaria. Estudios Sobre Educación, Pamplona, v. 30, p. 115-143, 2016.

MURILLO, Javier. El movimiento teórico-práctico de mejora de la escuela: algunas lecciones aprendidas para transformar los centros docentes. Revista Electrónica Iberoamericana sobre Calidad, Eficacia y Cambio en Educación, Madrid, v. 1, n. 2, p. 1-22.2003.

OCDE - Organización para Cooperación y Desarrollo Económico. Education at a glance 2011: OECD indicators. Paris: OCDE, 2011.

PERRY, Laura. Characteristics of equitable systems of education. European Education, London, v. 41, n. 1 , p. 79-100, 2009.

POPKEWITZ, Thomas. Paradigmas e ideología en la investigación educativa. Madrid: Mondadori. 1988.

PORTA, Luis; SILVA, Miriam. La investigación cualitativa: el análisis de contenido en la investigación educativa. 2003. Disponible en: <http://anthropostudio.com/wp-content/uploads/2015/04/PORTA-Luisy-SILVA-Miriam-2003.-La-investigaci\%C3\%B3n-cualitativa.-El-An\%C3\%A1lisis-de-Contenido-en-lainvestigaci\%C3\%B3n-educativa..pdf>. Acceso: 23 feb. 2015.

RITACCO, Maximiliano; AMORES; Francisco; MORAL; Cristina. El desarrollo de procesos de autoevaluación como capacidad del liderazgo pedagógico: un estudio en educación secundaria en Andalucía. Revista Iberoamericana de Evaluación Educativa, Madrid, v. 8, n. 2, p. 1-20, 2015.

RITACCO, Maximiliano; BOLIVAR,Antonio. Impacto del modelo español de dirección escolar en laidentidad profesional de los líderes escolares. Archivos Analíticos de Políticas Educativas, Arizona, v. 24, n. 119, p. 1-38, 2016.

ROWAN, Brian. Commitment and control: alternative strategies for the organizational designs of school. 
Review of Research in education, v. 16, p. 353-389, 1990.

SMITH, Jonathan; FLOWER, Paul; LARKIN, Michael. Interpretative phenomenological analysis: theory, method and research. Londres: Sage, 2009.

SPILLANE, James. Distributed leadership. London: Jossey-Bass, 2006.

STRAUSS, Anselm; CORBIN, Juliet. Bases de la investigación cualitativa: técnicas y procedimientos para desarrollar la teoría fundamentada. Colombia: Universidad de Antioquia, 2002.

VAILLANT, Denise. Liderazgo escolar, evolución de políticas y prácticas y mejora de la calidad educativa. In: UNESCO. Education for all 2000-2015: achievements and challenges. [S. I.]: Unesco, 2015. p. 1-27. Background paper prepared for the education for all global monitoring report 2015.

VAILLANT, Denise; RODRÍGUEZ, Eduardo. Prácticas de liderazgo para el aprendizaje en América Latina: un análisis a partir de PISA 2012. Ensaio, Rio de Janeiro, v. 24, n. 91, p. 253-274, 2016.

VALLÉS, Miguel. Entrevistas cualitativas: cuadernos metodológicos. Madrid: CIS, 2009.

WEINSTEIN, José; HERNÁNDEZ, Macarena. Birthpains: emerging school leadership policies in eight school systems of Latin America. International Journal of Leadership in Education, v. 19, n. 3, p. 1-23, 2015.

WERTZ, Frederick; CHARMAZ, Kathy; McMULLEN, Linda. Five ways of doing qualitative analysis: phenomenological psychology, grounded theory, discourse analysis, narrative research, and intuitive inquiry. New York: The Guilford Press, 2011.

Recibido en: 04.04.2016

Aprobado en: 06.12.2016

Maximiliano Ritacco es profesor ayudante doctor en el área de Teoría e Historia de la Educación de la Universidad de Zaragoza (España). Miembro de la Red Temática de Excelencia RILME (Red de Investigación de Liderazgo y Mejora de la Escuela), contando con varias publicaciones en esta línea de investigación.

Francisco Javier Amores es profesor ayudante doctor en el área de Didáctica y Organización Escolar (Departamento de Didáctica) de la Universidad de Cádiz (España). Miembro del Grupo de Investigación FORCE (HUM-386) y de la Red Temática de Excelencia RILME, contando con varias publicaciones en esta línea de investigación. 